\title{
Relation between Structure and Magnetic Properties of Microstructured $\mathrm{PrAlO}_{3}$
}

\author{
B. Andrzejewski ${ }^{a, *}$, D.A. PAWlak ${ }^{b}$, T. KLIMCZUK $^{c, d}$ AND S. TurCZYŃSKI ${ }^{b}$ \\ ${ }^{a}$ Institute of Molecular Physics, Polish Academy of Sciences, M. Smoluchowskiego 17, 60-179 Poznań, Poland \\ ${ }^{b}$ Institute of Electronic Materials Technology, Wólczynska 133, 01-919 Warsaw, Poland \\ ${ }^{c}$ Faculty of Applied Physics and Mathematics, Gdańsk University of Technology \\ G. Narutowicza 11/12, 80-952 Gdańsk, Poland \\ ${ }^{d}$ European Commission, Joint Research Centre, Institute for Transuranium Elements \\ Postfach 2340, Karlsruhe, D-76125 Germany
}

\begin{abstract}
The magnetic properties of both the praseodymium-aluminium perovskite $\mathrm{PrAlO}_{3}$ crystal and its microstructured version in the form of a $\mathrm{PrAlO}_{3}-\mathrm{PrAl}_{11} \mathrm{O}_{18}$ eutectic have been investigated. It is shown that $R \overline{3} c \rightarrow I m m a$ $205 \mathrm{~K}$ first-order and Imma $\rightarrow C 2 / m$ near $150 \mathrm{~K}$ second-order phase transitions in a $\mathrm{PrAlO}_{3}$ single crystal are suppressed after structuring the material and embedding it in a $\operatorname{PrAl}_{11} \mathrm{O}_{18}$ matrix. This behavior is related to the $\operatorname{PrAl}_{11} \mathrm{O}_{18}$ matrix, which mechanically hinders expansion of the microrods and in this way suppresses the phase transitions.
\end{abstract}

PACS: 64.60.Ej, 71.70.Ej, 75.50.-y, 75.70.Tj

\section{Introduction}

Recently, the active area of research are oxide-oxide self-organized eutectics manufactured with the aim of obtaining unknown in nature properties such as negative refractive index [1], cloaking [2], artificial magnetism [3], or giant dielectric constant [4]. The example of such a material is the praseodymium aluminate $\left(\mathrm{PrAlO}_{3}\right)$ praseodymium hexaaluminate $\left(\mathrm{PrAl}_{11} \mathrm{O}_{18}\right)$ eutectic, $\mathrm{PrAlO}_{3}-\mathrm{PrAl}_{11} \mathrm{O}_{18}$ [5] formed by $\mathrm{PrAlO}_{3}$ microrods spaced hexagonally in the $\mathrm{PrAl}_{11} \mathrm{O}_{18}$ matrix.

At room temperature the $\mathrm{PrAlO}_{3}$ crystal itself is a rhombohedrally-distorted perovskite characterised by space group $R \overline{3} c[6,7]$, but it undergoes a series of complex phase transitions as the temperature is changed: $C 2 / m$ (monoclinic) $\stackrel{150 \mathrm{~K}}{\longrightarrow}$ Imma (orthorhombic) $\stackrel{205 \mathrm{~K}}{\longrightarrow}$ $R \overline{3} c$ (rhombohedral) $\stackrel{1650 \mathrm{~K}(1770 \mathrm{~K})}{\longrightarrow} \operatorname{Pm} \overline{3} m$ (cubic) [7, 8]. The phase transitions at low temperatures [8,9] are a first-order one (discontinuous) at about $205 \mathrm{~K}$ and a second-order one (continuous) near $150 \mathrm{~K}$. On the other hand, at high temperatures the rhombohedral distortion is reduced, and above about $1770 \mathrm{~K}, \mathrm{PrAlO}_{3}$ exhibits the cubic structure of the ideal perovskite [8].

In the present work, we investigate the magnetic properties of a $\mathrm{PrAlO}_{3}$ crystals and compare them with the

* corresponding author; e-mail: and@ifmpan.poznan.pl properties of a $\operatorname{PrAlO}_{3}-\mathrm{PrAl}_{11} \mathrm{O}_{18}$ eutectic. As it will be shown, due to the influence of microstructure, some phase transitions of pure $\mathrm{PrAlO}_{3}$ are completely absent when it grows in the form of microrods hexagonally embedded in a matrix of $\operatorname{PrAl}_{11} \mathrm{O}_{18}$.

\section{The experiment}

The $\mathrm{PrAlO}_{3}$ single crystals with the stoichiometric composition have been obtained by the Czochralski method described elsewhere [10]. The $\mathrm{PrAlO}_{3}$ $\operatorname{PrAl}_{11} \mathrm{O}_{18}$ eutectics were grown by means of the micro-pulling down method [11]. The specification of the thermal system used and the growth conditions can be found in Ref. [12]. The eutectic crystals were seed-grown with a $\mathrm{YAlO}_{3}$ single crystal and $\mathrm{Al}_{2} \mathrm{O}_{3}, \mathrm{Pr}_{6} \mathrm{O}_{11}$ oxide powders (99.995\% purity) were used as starting materials.

The $\mathrm{PrAlO}_{3}-\mathrm{PrAl}_{11} \mathrm{O}_{18}$ eutectic rod obtained by the micro-pulling down method is presented in the inset to Fig. 1, whereas the main part shows eutectic microstructure composed of the $\mathrm{PrAlO}_{3}$ microfibers hexagonally placed in the $\mathrm{PrAl}_{11} \mathrm{O}_{18}$ matrix. The details of this microstructure strongly depends on the pulling rate - the cross-section of the $\mathrm{PrAlO}_{3}$ microrods and their distribution becomes more regular as the pulling rate increases. Simultaneously, the diameter of microrods decreases with the increase of the pulling rate.

Magnetization measurements were performed by means of Quantum Design MPMS system (SQUID). For 


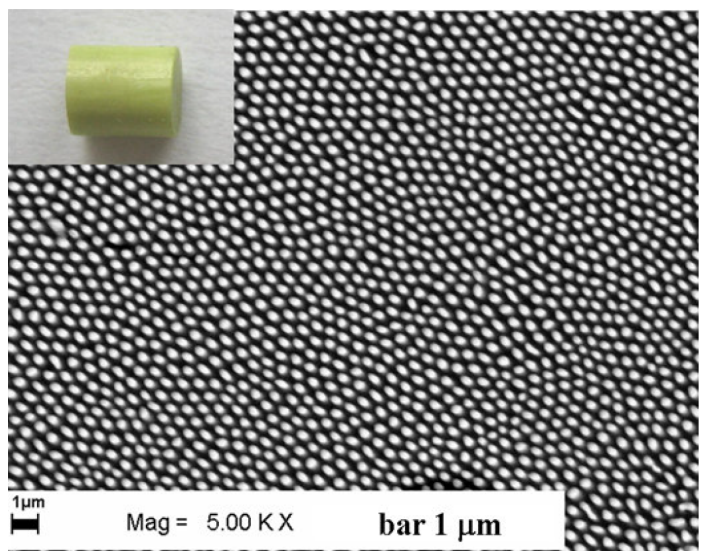

Fig. 1. The $\operatorname{PrAlO}_{3}-\mathrm{PrAl}_{11} \mathrm{O}_{18}$ eutectic: the rodlike microstructure of the eutectic; inset is the sample cut from an as-grown eutectic rod.

the measurements, a set of three $\mathrm{PrAlO}_{3}-\mathrm{PrAl}_{11} \mathrm{O}_{18}$ eutectic samples and one single $\mathrm{PrAlO}_{3}$ crystal for comparison were selected. To obtain proper dimensions for SQUID the eutectic samples were cut perpendicular to the crystal growth direction and perpendicular to $\mathrm{PrAlO}_{3}$ microrods. The $\mathrm{PrAlO}_{3}-\mathrm{PrAl}_{11} \mathrm{O}_{18}$ eutectic samples were as follows: sample " 0.3 " — pulled at the rate p.r. $=0.3 \mathrm{~mm} / \mathrm{min}$, mean radius of microrods $\langle r\rangle=1.35 \pm 0.01 \mu \mathrm{m}$; sample " 0.45 " - p.r. $=$ $0.45 \mathrm{~mm} / \mathrm{min},\langle r\rangle=1.06 \pm 0.01 \mu \mathrm{m}$; sample "5" - p.r. $=$ $5 \mathrm{~mm} / \mathrm{min},\langle r\rangle=0.39 \pm 0.01 \mu \mathrm{m} \mathrm{[5].} \mathrm{The} \mathrm{magnetic}$ field was applied parallel to the microrods. The $\mathrm{PrAlO}_{3}$ sample was cut perpendicular to crystal growth direction from the as-grown $\mathrm{PrAlO}_{3}$ crystal. The magnetic field was applied parallel to the growth direction.

\section{Results and discussion}

Figure 2 shows the results of susceptibility $\chi(T)$ measurements for $\mathrm{PrAlO}_{3}$ single crystal recorded for applied magnetic field $1 \mathrm{~T}$. The zero field cooling (ZFC) and field cooling (FC) $\chi(T)$ data mostly coincide, except the range from $\approx 140 \mathrm{~K}$ to $\approx 200 \mathrm{~K}$. This suggests that there are no irreversible magnetization processes related to, for example, domain reorientations [9, 13]. The step discontinuity in $\chi(T)$ at $205 \mathrm{~K}$ corresponds to the Imma $\rightarrow R \overline{3} c$ first-order structural transition whereas the change near $150 \mathrm{~K}$ is associated with a $C 2 / m \rightarrow I m m a$ second-order structural transition. Like in a few reports [13], we have not observed here both distinct structural transitions in a single measuring procedure i.e. ZFC or FC. This behavior is caused by internal strains which can smear the transition or even change it from first-order to second-order like or opposite [13]. This is really observed in Fig. 2. Below $40 \mathrm{~K}$ the susceptibility is practically constant because of the Van Vleck temperature-independent paramagnetism [14].

Obviously, the transitions discussed here are structural and rely on rotation of the $\mathrm{AlO}_{6}$ octahedra about

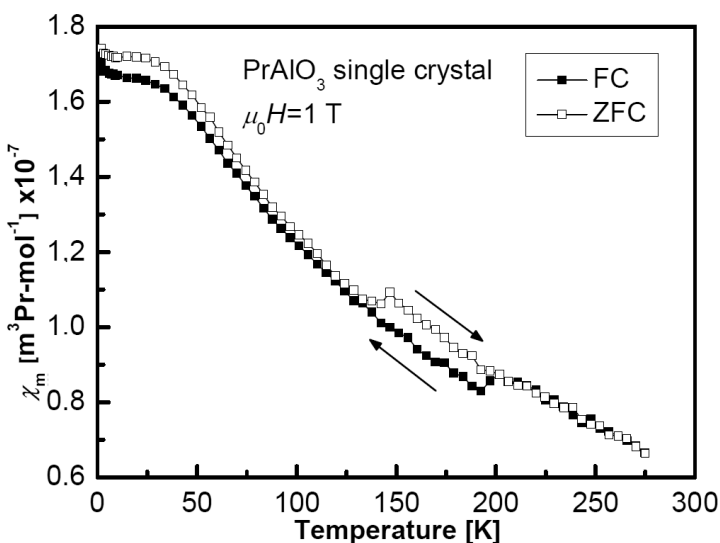

Fig. 2. Magnetic properties of $\mathrm{PrAlO}_{3}$ single crystal recorded for $\mathrm{ZFC}$ and $\mathrm{FC}$ mode for applied magnetic field $\mu_{0} H=1 \mathrm{~T}$.

corresponding crystallographic axes. However, they can be monitored with magnetometric measurements due to the strong coupling of $\mathrm{Pr}^{3+}$ ion levels to the crystal lattice, which leads to changes in the magnetic moment of praseodymium when the lattice distorts during the transitions.

The main part in Fig. 3 presents the temperature dependences of ZFC susceptibility $\chi(T)$ for three selected $\mathrm{PrAlO}_{3}-\mathrm{PrAl}_{11} \mathrm{O}_{18}$ eutectic samples. The FC susceptibility curves, coincides well to ZFC ones and are omitted here for clarity of the picture. Unlike the behavior of single crystals of $\mathrm{PrAlO}_{3}$, in these eutectics the $205 \mathrm{~K}$ transition is completely absent. Week trace of $150 \mathrm{~K}$ phase transition appears in the inverse of susceptibility $1 / \chi(T)$, only (inset to Fig. 3). Moreover, instead of a Van Vleck regime of constant magnetization observed in $\mathrm{PrAlO}_{3}$, in the eutectic samples substantial increase of susceptibility appears in low temperature.

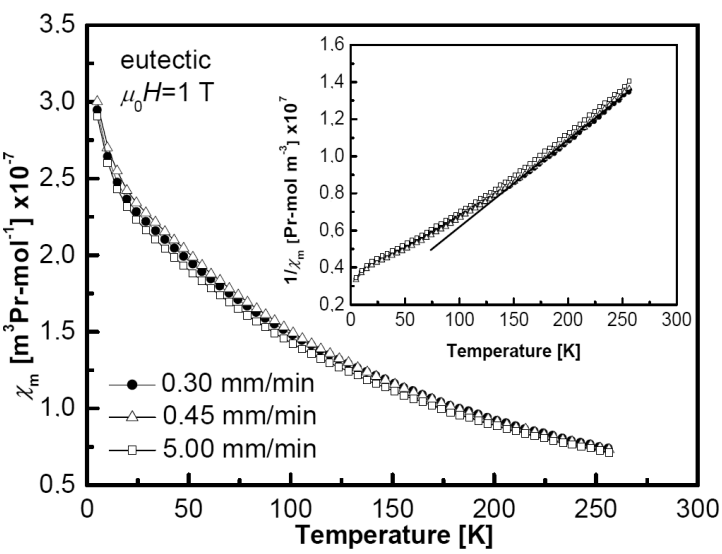

Fig. 3. ZFC susceptibility vs. temperature, $\chi(T)$, of $\mathrm{PrAlO}_{3}-\mathrm{PrAl}_{11} \mathrm{O}_{18}$ eutectics grown with pulling rates; $0.3 \mathrm{~mm} / \mathrm{min}, 0.45 \mathrm{~mm} / \mathrm{min}$ and $5 \mathrm{~mm} / \mathrm{min}$. The inset shows the inverse of susceptibility $1 / \chi(T)$. The applied magnetic field was $\mu_{0} H=1 \mathrm{~T}$. 
The exact interpretation of these data is difficult because, besides $\mathrm{PrAlO}_{3}$, the eutectic sample also contains a $\operatorname{PrAl}_{11} \mathrm{O}_{18}$ phase, whose magnetic properties are completely unknown. Therefore it is impossible to separate the magnetic contribution of the $\mathrm{PrAlO}_{3}$ microrods out of the total signal and to compare it with magnetic properties of single $\mathrm{PrAlO}_{3}$ crystals. However one can expect that the strong increase in the magnetic susceptibility $\chi(T)$ of the eutectics at low temperatures, which probably mask the constant Van Vleck susceptibility of $\mathrm{PrAlO}_{3}$ microrods, is due to a strong paramagnetic moment of $\mathrm{PrAl}_{11} \mathrm{O}_{18}$ matrix.

The absence of a high-temperature transition at $205 \mathrm{~K}$ in eutectics is probably related to their microstructure which consists of hexagonal network of $\mathrm{PrAlO}_{3}$ microrods embedded in $\mathrm{PrAl}_{11} \mathrm{O}_{18}$ matrix phase. The presence of matrix stabilize the crystallographic structure of $\mathrm{PrAlO}_{3}$ microrods because it hinders lateral expansion of microrods due to changes in lattice constants of $\mathrm{PrAlO}_{3}$ phase associated with the structural transitions. It can also pin the $\mathrm{PrAlO}_{3}$ microstructures to the $\mathrm{PrAl}_{11} \mathrm{O}_{18}$ matrix at the microrods/matrix interface. The $\operatorname{PrAl}_{11} \mathrm{O}_{18}$ matrix surrounding the $\mathrm{PrAlO}_{3}$ microrods can inhibit the relatively weak thermal changes of their dimensions at $R \overline{3} c \rightarrow$ Imma $($ Imma $\rightarrow R \overline{3} c)$ phase transition near $205 \mathrm{~K}$. This is however, impossible at the phase transition near $150 \mathrm{~K}($ Imma $\rightarrow C 2 / m)$, where the changes in dimensions of microrods are much more substantial. Therefore, in the eutectics, the first order $205 \mathrm{~K}$ phase transition is completely suppressed but there are still visible small traces of the second-order $150 \mathrm{~K}$ transition in the $1 / \chi(T)$ plot.

The size of microrods, and thus the surface-to-rod volume ratio, does not influence the transitions significantly. This gives evidence that the mechanical effect of the $\mathrm{PrAl}_{11} \mathrm{O}_{18}$ matrix dominates over the interfacial pinning of $\mathrm{PrAlO}_{3}$ and is responsible for the reduction of magnitude of phase transitions in the eutectics.

\section{Conclusions}

The $\mathrm{PrAlO}_{3}$ crystal and $\mathrm{PrAlO}_{3}-\mathrm{PrAl}_{11} \mathrm{O}_{18}$ eutectic composed of $\mathrm{PrAlO}_{3}$ microrods embedded in $\mathrm{PrAl}_{11} \mathrm{O}_{18}$ matrix were successfully grown by the Czochralski and the micro-pulling down method, respectively. In the magnetic measurements, we have observed both Imma $\rightarrow C 2 / m$ (at $150 \mathrm{~K}$ ) second-order and $R \overline{3} c \rightarrow$ Imma (at $205 \mathrm{~K}$ ) first-order structural phase transitions in $\mathrm{PrAlO}_{3}$ single crystal. As opposed to single crystals $\mathrm{PrAlO}_{3}-\mathrm{PrAl}_{11} \mathrm{O}_{18}$ eutectics exhibit lack of high- -temperature transition at $205 \mathrm{~K}$ and only very weak trace of transition at $150 \mathrm{~K}$. This behavior is explained assuming that $\operatorname{PrAl}_{11} \mathrm{O}_{18}$ matrix prevents lateral expansion of microrods during the structural transitions and in this way stabilizes the crystallographic structure of $\mathrm{PaAlO}_{3}$ phase.

\section{Acknowledgments}

D.A.P. and B.A. thank the Ministry of Scientific Research and Information Technology of Poland (N515028 $31 / 1103$ ) for partial support for conduct of the research and magnetometric measurements. The authors thank to the FP7 NMP ENSEMBLE project (GA NMP4-SL-2008-213669) for partially supporting of the research.

\section{References}

[1] D.R. Smith, J.B. Pendry, M.C.K. Wilthshire, Science 305, 788 (2004).

[2] J.B. Pendry, D. Schurig, D.R. Smith, Science 312, 1780 (2006).

[3] J.B. Pendry, Science 285, 1687 (1999).

[4] C. Pecharroman, F. Esteban-Betegon, J.F. Bartolome, S. Lopez-Esteban, J.S. Moya, Adv. Mater. 13, 1541 (2001).

[5] D.A. Pawlak, K. Kołodziejak, K. Różniatowski, R. Diduszko, M. Kaczkan, M. Malinowski, M. Piersa, J. Kisielewski, T. Łukasiewicz, Cryst. Growth Des. 8, 1243 (2008)

[6] A.M. Glazer, Acta Crystallogr. B 28, 3384 (1972).

[7] C.J. Howard, B.J. Kennedy, B.C. Chakoumakos, J. Phys., Condens. Matter. 12, 349 (2000).

[8] C.J. Howard, H. Stokes, Acta Crystallogr. B: Struct. Sci. 54, 782 (1998).

[9] E. Cohen, L.A. Risberg, W.A. Nordland, R.D. Burbank, R.C. Sherwood, L.G. Van Uitert, Phys. Rev. 186, 476 (1969).

[10] J. Czochralski, Z. Phys. Chem. 92, 219 (1918).

[11] D.H. Yoon, I. Yonenaga, N. Ohnishi, T.J. Fukuda, Cryst. Growth 142, 339 (1994).

[12] K. Kołodziejak, S. Turczyński, R. Diduszko, L. Klimek, D.A. Pawlak, Optoelectron. Rev. 14, 203 (2006).

[13] M.D. Sturge, E. Cohen, L.G. Van Uitert, Phys. Rev. B 11, 4768 (1975).

[14] A.M. Lejus, J.C. Bernier, R.J. Collongues, J. Solid State Chem. 16, 349 (1976). 